\title{
FLOW-INVARIANCE PROPERTIES \\ FOR A CLASS OF DISCRETE-TIME \\ NONLINEAR UNCERTAIN SYSTEMS
}

\author{
Laurentiu Marinovici and Octavian Pastravanu \\ Department of Automatic Control and Industrial Informatics \\ Technical University "Gh. Asachi" of Iasi \\ Blvd. Mangeron 53A, 6600 Iasi, Romania \\ Phone: +40-32-230751, Fax: +40-32-214290 \\ Imarinovici@ac.tuiasi.ro \\ opastrav@ac.tuiasi.ro
}

\begin{abstract}
The family of time-dependent rectangular sets, flow invariant with respect to a class of discrete-time nonlinear uncertain systems, is studied. The invariance results are further used for dealing with a special type of asymptotic stability, called componentwise asymptotic stability (CWAS), which can be characterized by difference inequalities. The particularisation of the CWAS conditions for exponential type timedependence yields the stronger property of componentwise exponential asymptotic stability (CWEAS), that is proven equivalent to some algebraic inequalities.
\end{abstract}

Keywords: Flow-invariant sets, discrete-time systems, nonlinear systems, uncertain systems.

AMS Subject Classification: 93C10, 93D20, 93C55, 93C41

\section{Introduction}

Using the powerful tool offered by the flow-invariance theory, a componentwise refinement is envisaged in approaching the dynamics of a class of discrete-time nonlinear systems with uncertainties. Such a refinement, by individually monitoring each state variable, is able to reveal some important properties of the trajectories around the equilibrium points, which remain hidden within the standard framework of stability analysis. The usage of flow-invariance concepts for a componentwise exploration of the dynamical system behavior appeared in mid eighties

The original version of this chapter was revised: The copyright line was incorrect. This has been corrected. The Erratum to this chapter is available at DOI: 10.1007/978-0-387-35690-7_44 
in Pavel's and Voicu's works ([4]), ([5]), ([6]), ([7]), focusing exclusively on the continuous-time case. The discrete-time case was addressed later in papers, such as ([1]) for 1-D and 2-D linear systems and ([2]) for linear systems with uncertainties. All the above cited works pointed out that the componentwise investigation naturally leads to particular types of asymptotic stability, namely the componentwise asymptotic stability (CWAS) and componentwise exponential asymptotic stability (CWEAS), as initially introduced in ([5]), ([6]).

The class of nonlinear uncertain systems studied in the current paper may be roughly regarded as a discrete-time counterpart of the continuoustime dynamics discussed in ([3]). In spite of the similarities occurring at the first glance between the mathematical description of the continuoustime and discrete-time cases, this deeper insight emphasizes noticeable differences, which are commented throughout the text. Our material is structured as follows. Section 2 deals with the existence of timedependent rectangular sets (TDRSs), which are flow-invariant (FI) with respect to a given discretë-time nonlinear uncertain system (DTNUS). Sections 3 and 4, by using the FI results, analyse CWAS, and respectively, CWEAS of the equilibrium point (EP) $\{0\}$. Some concluding remarks, including comparisons with the continuous-time case in ([3]) are formulated in Section 5. Since the complete proofs of most results are laborious this version of the text limits them to some basic explanation.

\section{The Family of TDRSs with FI Properties}

Consider the class of discrete-time nonlinear uncertain systems (DTNUSs) defined as:

$$
\begin{aligned}
\mathbf{x}(t+1) & =\mathbf{f}(\mathbf{x}(t)), \mathbf{x} \in \mathbb{R}^{n}, \mathbf{x}\left(t_{0}\right)=\mathbf{x}_{0}, t \in \mathbb{Z}_{+} \\
f_{i}(\mathbf{x}(t)) & =\sum_{j=1}^{n} a_{i j} x_{j}^{p_{i j}}(t), p_{i j} \in \mathbb{N}, i=\overline{1, n}
\end{aligned}
$$

with interval-type coefficients:

$$
a_{i j}^{-} \leq a_{i j} \leq a_{i j}^{+}, i, j=\overline{1, n} .
$$

Also consider the $n$-valued vector function $\mathbf{v}(t): \mathbb{Z}_{+} \rightarrow \mathbb{R}^{n}$ with positive components $v_{i}(t)>0, i=\overline{1, n}$. Using these $v_{i}(t)>0, i=\overline{1, n}$ define the time-dependent rectangular set (TDRS).

$$
\mathbf{H}_{v}(t)=\left[-v_{1}(t), v_{1}(t)\right] \times \ldots \times\left[-v_{n}(t), v_{n}(t)\right],
$$


where $[] \times,[$,$] denotes the Cartesian product.$

Let us explore the free response of DTNUS (1) along the lines of the componentwise constrained evolution of the state trajectories induced by the concept of flow-invariance (FI).

Definition 1 TDRS (3) is called flow-invariant (FI) with respect to (w.r.t.) DTNUS (1) if for any initial condition $\mathbf{x}\left(t_{0}\right)=\mathbf{x}_{0} \in \mathbf{H}_{v}\left(t_{0}\right)$, the corresponding state trajectory $\mathbf{x}(t)=\mathbf{x}\left(t ; t_{0}, \mathbf{x}_{0}\right)$ remains (for all possible values resulting from the interval-type coefficients) inside $\mathbf{H}_{v}(t)$, for $t \in\left[t_{0}, \infty\right)$, i.e.

$$
\begin{aligned}
\forall \mathbf{x}\left(t_{0}\right) & =\mathbf{x}_{0} \in \mathbf{H}_{v}\left(t_{0}\right): \\
\mathbf{x}(t) & =\mathbf{x}\left(t ; t_{0}, \mathbf{x}_{0}\right) \in \mathbf{H}_{v}(t), t \in\left[t_{0}, \infty\right)
\end{aligned}
$$

Theorem 1 TDRS (3) is FI w.r.t. DTNUS (1) iff the following vector inequalities hold for $t \in\left[t_{0}, \infty\right)$ :

$$
\begin{aligned}
& \mathbf{v}(t+1) \geq \overline{\mathbf{g}}(\mathbf{v}(t)) ; \bar{g}_{i}(\mathbf{v}(t))=\sum_{j=1}^{n} \bar{c}_{i j} v_{j}^{p_{i j}}(t), i=\overline{1, n} \\
& \mathbf{v}(t+1) \geq \widetilde{\mathbf{g}}(\mathbf{v}(t)) ; \tilde{g}_{i}(\mathbf{v}(t))=\sum_{j=1}^{n} \widetilde{c}_{i j} v_{j}^{p_{i j}}(t), i=\overline{1, n}
\end{aligned}
$$

where $\bar{c}_{i j}, \tilde{c}_{i j}$ have unique values, derived from the interval-type coeffcients $a_{i j}$ of DTNUS (1) as follows:

$$
\begin{aligned}
& \bar{c}_{i j}=\left\{\begin{array}{l}
\max \left\{\left|a_{i j}^{-}\right|,\left|a_{i j}^{+}\right|\right\}, \text {if } p_{i j} \text { odd } \\
\max \left\{0, a_{i j}^{+}\right\}, \text {if } p_{i j} \text { even }
\end{array},\right. \\
& \tilde{c}_{i j}=\left\{\begin{array}{l}
\max \left\{\left|a_{i j}^{-}\right|,\left|a_{i j}^{+}\right|\right\}, \text {if } p_{i j} \text { odd } \\
\max \left\{0,-a_{i j}^{-}\right\}, \text {if } p_{i j} \text { even }
\end{array} .\right.
\end{aligned}
$$

Proof. It results by expressing the bounds of the right-hand side of (1) in terms of $a_{i j}^{-}, a_{i j}^{+}, i, j=\overline{1, n}$ in (2) and $v_{i}(t), i=\overline{1, n}$, in (3).

Theorem 2 There exist TDRSs (3) which are FI w.r.t. DTNUS (1) iff there exist positive solutions (PSs) $y_{i}(t)>0, i=\overline{1, n}$ for the following difference inequalities (DIs):

$$
\begin{aligned}
& \mathbf{y}(t+1) \geq \overline{\mathbf{g}}(\mathbf{y}(t)) \\
& \mathbf{y}(t+1) \geq \widetilde{\mathbf{g}}(\mathbf{y}(t)) .
\end{aligned}
$$


Proof. On the basis of Definition 1 and Theorem 1, any positive solution $y_{i}(t)>0, i=\overline{1, n}$ of the inequality system (7-a), (7-b) defines a flow invariant TDRS (3).

Remark 1 The algorithm for constructing the coefficients (6-a) and (6-b) of DIs (7-a) and (7-b) differs from its continuous-time counterpart studied in ([3]), yielding only positive values for these coefficients as also occurring for discrete-time linear systems, e.g. ([1]), ([2]).

Theorem 3 There exist TDRSs (3) which are FI w.r.t. DTNUS (1) iff there exist PSs $y_{i}(t)>0, i=\overline{1, n}$ for the following $D I$ :

$$
\mathbf{y}(t+1) \geq \mathbf{g}(\mathbf{y}(t)) ; \mathbf{g}: \mathbb{R}^{n} \rightarrow \mathbb{R}^{n}, g_{i}(\mathbf{y})=\max _{\mathbf{y} \in \mathbb{R}^{n}}\left\{\bar{g}_{i}(\mathbf{y}), \tilde{g}_{i}(\mathbf{y})\right\}, i=\overline{1, n} .
$$

Proof. Inequality (8) replaces the two inequalities (7-a) and (7-b) from Theorem 2 in an equivalent manner.

In order to investigate the family of TDRSs, which are FI w.r.t. a given DTNUS, we will first focus on some relevant characteristics of the PSs of the inequality (8), since Theorem 3 emphasizes a bijective link between the two types of mathematical objects. We start with the qualitative exploration of the solution of the following difference equation (DE):

$$
\mathbf{z}(t+1)=\mathbf{g}(\mathbf{z}(t)), t \in \mathbb{Z}_{+}
$$

which is obtained from DI (8) by replacing " $\geq$ " with $"="$.

Theorem 4 For any $t_{0} \in \mathbb{Z}_{+}$and any positive initial condition $\mathbf{z}\left(t_{0}\right)=$ $\mathbf{z}_{0}>0$, the unique solution $\mathbf{z}(t)=\mathbf{z}\left(t ; t_{0}, \mathbf{z}_{0}\right)$ of $D E(9)$ remains positive for any $t \in\left[t_{0}, \infty\right)$.

Proof. According to the definition of $\bar{g}_{i}$ and $\tilde{g}_{i}$ in (5-a) and (5-b), all the coefficients $\bar{c}_{i j}, \widetilde{c}_{i j}, i, j=\overline{1, n}$ are non-negative, and thus, for any $t_{0} \in \mathbb{Z}_{+}$and any positive initial condition $\mathbf{z}\left(t_{0}\right)=\mathbf{z}_{0}>0$, since $g_{i}(\mathbf{z})=$ $\max _{\mathbf{z} \in \mathbb{R}^{n}}\left\{\bar{g}_{i}(\mathbf{z}), \widetilde{g}_{i}(\mathbf{z})\right\}$, the solution $\mathbf{z}(t)=\mathbf{z}\left(t ; t_{0}, \mathbf{z}_{0}\right)$ of DE (9) remains positive for any $t \in\left[t_{0}, \infty\right)$.

One can easily see that Theorem 4 guarantees the existence of PSs for DI (8) in the particular case when " $\geq "$ is replaced by " = ". However DI (8) might have PSs, which do not satisfy DE (9) and therefore, a connection between the PSs of DI (8) and the PSs of DE (9) must be further established.

Theorem 5 Let $\mathbf{y}(t)>0$ be an arbitrary PS of DI (8), with $t \in\left[t_{0}, \infty\right)$, $t_{0} \in \mathbb{Z}_{+}$. Denote by $\mathbf{z}(t)$ an arbitrary $P S$ of $D E(9)$, corresponding to 
an initial condition $\mathbf{z}\left(t_{0}\right)$, which satisfies the componentwise inequality $0<\mathbf{z}\left(t_{0}\right) \leq \mathbf{y}\left(t_{0}\right)$. Denote by $\mathbf{z}^{*}(t)$ the unique PS of DE (9) corresponding to the initial condition taken by $\mathbf{y}(t)$, i.e. $\mathbf{z}^{*}\left(t_{0}\right) \equiv \mathbf{y}\left(t_{0}\right)$. For $t \in\left[t_{0}, \infty\right)$ the following inequalities hold: $0<\mathbf{z}(t) \leq \mathbf{z}^{*}(t) \leq \mathbf{y}(t)$.

Proof. The fulfillment of the inequality $0<\mathbf{z}(t)$ for $0<\mathbf{z}\left(t_{0}\right)$ is guaranteed by Theorem 4 .

Since $0<\mathbf{z}\left(t_{0}\right) \leq \mathbf{z}^{*}\left(t_{0}\right) \equiv \mathbf{y}\left(t_{0}\right)$, it results that: $0<g_{i}\left(\mathbf{z}\left(t_{0}\right)\right) \leq$ $g_{i}\left(\mathbf{z}^{*}\left(t_{0}\right)\right)=g_{i}\left(\mathbf{y}\left(t_{0}\right)\right), i=\overline{1, n}$, and so $0<\mathbf{z}\left(t_{0}+1\right) \leq \mathbf{z}^{*}\left(t_{0}+1\right) \leq$ $\mathbf{y}\left(t_{0}+1\right)$.

By mathematical induction, one can easily see that the inequalities at time $(t-1), t \in \mathbb{Z}_{+}$imply the truthfulness of the same inequalities at time $(t), t \in \mathbb{Z}_{+}$.

Remark 2 Unlike the continuous-time case studied in ([3]), where the -FI property might be restricted to a finite-time horizon $\left[t_{0}, T\right)$, the existence of FI w.r.t. DTNUS (1) is guaranteed for an infinite-time horizon $\left[t_{0}, \infty\right)$.

Theorem 6 If $\mathbf{H}_{\mathbf{y}}(t), \mathbf{H}_{\mathbf{z}^{*}}(t)$ and $\mathbf{H}_{\mathbf{z}}(t)$ denote three TDRSs, FI w.r.t. DTNUS (1), generated by the following three types of PSs of DI (8): $\mathbf{y}(t)$ - arbitrary PS of DI (8); $\mathbf{z}^{*}(t)$ - unique PS of DE (9), with $\mathbf{z}^{*}\left(t_{0}\right) \equiv \mathbf{y}\left(t_{0}\right) ; \mathbf{z}(t)$ - arbitrary PS of DE (9), with $\mathbf{z}\left(t_{0}\right) \leq \mathbf{y}\left(t_{0}\right)$, then:

$$
\mathbf{H}_{\mathbf{z}}(t) \subseteq \mathbf{H}_{\mathbf{z}^{*}}(t) \subseteq \mathbf{H}_{\mathbf{y}}(t), \forall t \in\left[t_{0}, \infty\right)
$$

Proof. It results from Theorems 5 and 3 and Definition 1.

Given a TDRS, which is FI w.r.t. DTNUS (1), there can be formulated a condition for the existence of other TDRSs, strictly included in the former one, which are also FI w.r.t. DTNUS (1).

Theorem $\mathbf{7}$ Denote by $\mathbf{H}_{\mathbf{y}}(t)$ a TDRS, FI w.r.t. DTNUS (1) for $t \in$ $\left[t_{0}, \infty\right)$. If there exist $\mathrm{n}$ functions $\delta_{i}(t)$, non-decreasing, positive and subunitary $0<\delta_{i}(t)<1, i=\overline{1, n}, t \in \mathbb{Z}_{+}$, such that:

$$
\mathbf{g}(\boldsymbol{\Delta}(t) \mathbf{y}(t)) \leq \boldsymbol{\Delta}(t) \mathbf{g}(\mathbf{y}(t)) ; \boldsymbol{\Delta}(t)=\operatorname{diag}\left\{\delta_{1}, \ldots, \delta_{n}\right\},
$$

then the TDRS $\mathbf{H}_{\mathbf{\Delta} \mathbf{y}}(t)$, generated by the vector function $\boldsymbol{\Delta}(t) \mathbf{y}(t)$ is also FI w.r.t. DTNUS (1) and

$$
\mathbf{H}_{\Delta \mathbf{y}}(t) \subset \mathbf{H}_{\mathbf{y}}(t), t \in\left[t_{0}, \infty\right) .
$$

Proof. One can show that $\boldsymbol{\Delta}(t) \mathbf{y}(t)$ is a PS of DI (8) and, then, applying Theorem 3 and Definition 1 , together with $0<\delta_{i}(t)<1$, completes the proof. 
Remark 3 Functions $\delta_{i}(t)$ can be chosen as positive, subunitary constants, case in which the resulting $\mathrm{TDRS} \mathbf{H}_{\boldsymbol{\Delta y}}(t)$ is homotetic with $\mathbf{H}_{\mathbf{y}}(t)$, taking different transformation factors for each component. When all $\delta_{i}(t), i=\overline{1, n}$ are equal to the same positive, subunitary constant, the transformation factors are identical for all the components.

The next step in refining the conditions imposed to the TDRS FI w.r.t. DTNUS (1) aims to force the boundedness property by adding a supplementary request for the time-dependence of TDRS, namely to approach $\{0\}$ for $t \rightarrow \infty$. Thus, the concept of FI induces a particular type of asymptotic stability (AS) for the equilibrium point (EP) $\{0\}$ of DTNUS (1), (stronger than the standard concept based on vector norms in $\mathbb{R}^{n}$ ), which is going to be separately studied in the following section.

\section{Componentwise Asymptotic Stability}

Let $\mathbf{v}(t):\left[t_{0}, \infty\right) \rightarrow \mathbb{R}^{n}$ be a vector function with $v_{i}(t)>0, i=\overline{1, n}$ (as considered in Definition 1, which introduces the FI concept) and suppose that $\mathbf{v}(t)$ also has the property:

$$
\lim _{t \rightarrow \infty} \mathbf{v}(t)=0 .
$$

Definition 2 EP $\{0\}$ of DTNUS (1) is called componentwise asymptotically stable w.r.t. $\mathbf{v}(t)\left(\mathrm{CWAS}_{\mathbf{v}}\right)$ if for any $\mathbf{x}\left(t_{0}\right)=\mathbf{x}_{0}$ with $\left|\mathbf{x}\left(t_{0}\right)\right| \leq$ $\mathbf{v}\left(t_{0}\right)$, the following inequality holds: $|\mathbf{x}(t)| \leq \mathbf{v}(t), t \in\left[t_{0}, \infty\right)$.

Remark 4 Definition 2 can be restated in terms of FI, by taking Definition 1, supplemented with condition (12) for the behavior at the infinity.

On the light of the above remark, the results presented in Theorems 2 and 3 can be immediately transformed to characterize CWAS $\mathrm{Cf}_{\mathbf{v}} \mathrm{EP}$ $\{0\}$ of DTNUS (1), yielding the following two theorems.

Theorem $8 E P\{0\}$ of DTNUS (1) is CWAS $S_{\mathbf{v}}$ iff there exist common PSs $\mathbf{v}(t)>0$ for DIs (7-a) and (7-b), with $\lim _{t \rightarrow \infty} \mathbf{v}(t)=0$.

Proof. It is a direct consequence of Theorem 2 for the particular case of TDRSs meeting condition (12).

Theorem $9 E P\{0\}$ of DTNUS (1) is CWAS $S_{\mathbf{v}}$ iff there exist PSs $\mathbf{v}(t)$ for $D I(8)$, with $\lim _{t \rightarrow \infty} \mathbf{v}(t)=0$.

Proof. It is a direct consequence of Theorem 3 in the particular case of TDRSs meeting condition (12). 
The boundedness of TDRSs on $\left[t_{0}, \infty\right)$ together with the requirement (12) introduces some restrictions for the exponents $p_{i i}$ and the intervaltype coefficients $a_{i i}$ of DTNUS (1).

Theorem 10 A necessary condition for EP $\{0\}$ of DTNUS (1) to be $C W A S_{\mathbf{v}}$ is that

$$
\max \left\{\left|a_{i i}^{-}\right|,\left|a_{i i}^{+}\right|\right\}\left(v_{i}\left(t_{0}\right)\right)^{p_{i i}-1}<1, i=\overline{1, n}
$$

where $\mathbf{v}\left(t_{0}\right)$ denotes the initial value for the vector function $\mathbf{v}(t)$.

Proof. It results from the fact that the solutions of DEs $v_{i}(t+1)=$ $\widehat{c}_{i i} v_{i}^{p_{i i}}(t), \widehat{c}_{i i}=\max \left\{\bar{c}_{i i}, \widetilde{c}_{i i}\right\}, i=\overline{1, n}$ should converge to $\{0\}$.

Remark 5 Unlike the necessary condition formulated by Theorem 9 in ([3]) requiring $p_{i i}$ - odd and $a_{i i}^{+}<0, i=\overline{1, n}$, the necessary condition given above does not restrict the integer values of the exponents $p_{i i}$, but it emphasizes the role of the initial values for the vector $\mathbf{v}(t)$ used in Definition 2 of CWAS. The lack of a complete similarity between the continuous-time and discrete-time cases is a consequence of the fact that DTNUS (1) cannot be obtained by the discretization of the continuoustime system considered in ([3]).

Remark 6 The proof of our Theorem 10 shows that by the replacement of " <" by " " in inequalities (13), a necessary condition is obtained for the boundedness of TDRS (3) (without meeting the convergence condition (12) of $\mathbf{v}(t)$ used in Definition 2). This boundedness condition may be regarded as corresponding to the necessary condition in the continuous-time case, given by Theorem 6 in ([3]).

In order to develop a refined interpretation of the result stated in Theorem 9 , it is of great interest to resume the qualitative analysis of the solutions of DI (8) and DE (9).

Theorem 11 Consider an arbitrary $P S \mathbf{y}(t)>0$ of $D I(8)$, with $t \in$ $\left[t_{0}, \infty\right)$, and the initial condition $\mathbf{y}\left(t_{0}\right)$ satisfying restriction (13) for all $i=\overline{1, n}$. If $\mathbf{z}(t)$ denotes an arbitrary solution of $D E(9)$ corresponding to the initial condition $\mathbf{z}\left(t_{0}\right)$ satisfying: $-\mathbf{y}\left(t_{0}\right) \leq \mathbf{z}\left(t_{0}\right) \leq \mathbf{y}\left(t_{0}\right)$, then the following inequality holds for any $t \in\left[t_{0}, \infty\right)$

$$
-\mathbf{y}(t) \leq \mathbf{z}(t) \leq \mathbf{y}(t) .
$$

Proof. Starting from the componentwise initial restrictions $-y_{i}\left(t_{0}\right) \leq$ $z_{i}\left(t_{0}\right) \leq y_{i}\left(t_{0}\right), t_{0} \in \mathbb{Z}_{+}$, it is easy to see that $-\mathbf{g}\left(\mathbf{y}\left(t_{0}\right)\right) \leq \mathbf{g}\left(\mathbf{z}\left(t_{0}\right)\right) \leq$ 
$\mathbf{g}\left(\mathbf{y}\left(t_{0}\right)\right)$. This former inequality leads to: $-\mathbf{y}\left(t_{0}+1\right) \leq \mathbf{z}\left(t_{0}+1\right) \leq$ $\mathbf{y}\left(t_{0}+1\right)$. By induction it is proven that inequality (14) holds for any $t \in\left[t_{0}, \infty\right)$.

Theorem 11 creates a deeper insight into the topology of the solutions (not only positive) of $\mathrm{DE}(9)$ in the vicinity of $\mathrm{EP}\{0\}$, which permits revealing the link between condition (12) and the nature of EP $\{0\}$ for $\mathrm{DE}(9)$.

Theorem $12 E P\{0\}$ of DTNUS (1) is $C W A S_{\mathbf{v}}$ iff $E P\{0\}$ of $D E(9)$ is $A S$.

Proof. Sufficiency. One can take a positive initial condition for DE (9) such that, according to Theorem 4, the corresponding solution is positive and also meets condition (12). Then one uses Theorem 9. Necessity. DI (8) has PSs meeting condition (12) and applying Theorem 11 one can prove the AS of $\mathrm{EP}\{0\}$ of DE (9).

Remark 7 It is obvious that the concept of $\mathrm{CWAS}_{\mathbf{v}}$ for EP $\{0\}$ of DTNUS (1) is not equivalent to the standard AS. If EP $\{0\}$ of DTNUS (1) is $\mathrm{CWAS}_{\mathbf{v}}$ then it is AS, but the converse statement is not true. However, Theorem 12 can be used as a sufficient condition for approaching the standard problem of AS of DTNUS (1), where the presence of uncertainties (expressed by interval-type coefficients) makes rather difficult the usage of classical procedures.

For practice, it is hard to handle DE (9) in order to check its AS, analytically. A more attractive approach is to just find a sufficient condition for $\mathrm{CWAS}_{\mathbf{v}}$ based on an operator with a more tractable form than $\mathbf{g}$ in $\mathrm{DE}(9)$.

Theorem 13 Consider the DE:

$$
\mathbf{z}(t+1)=\widehat{\mathbf{g}}(\mathbf{z}(t)) ; \widehat{g}_{i}(\mathbf{z})=\sum_{j=1}^{n} \widehat{c}_{i j} z_{j}^{p_{i j}}, i=\overline{1, n},
$$

where the coefficients $\widehat{c}_{i j}$ are defined by: $\widehat{c}_{i j}=\max \left\{\bar{c}_{i j}, \widetilde{c}_{i j}\right\}, i, j=\overline{1, n}$.

(i) If $E P\{0\}$ is $A S$ for DE (15), then $E P\{0\}$ is CWAS for DTNUS (1).

(ii) In the particular case when the interval-type coefficients $a_{i j}$ of DTNUS (1) satisfy the inequalities given bellow, for each $i, i=\overline{1, n}$ :

$$
\text { IF } p_{i j} \text {-even THEN }\left(a_{i j}^{+} \geq-a_{i j}^{-} \text {for all } j \text { OR } a_{i j}^{+} \leq-a_{i j}^{-} \text {for all } j\right) \text {, }
$$

the sufficient condition stated at (i) is also necessary for $E P\{0\}$ of DT$N U S$ (1) to be $C W A S_{\mathrm{v}}$. 
Proof. (i) results from the fact that any PS of DE (15) is also a PS of DI (8). (ii) Whenever (16) is true, one has $\mathbf{g}(\mathbf{z})=\widehat{\mathbf{g}}(\mathbf{z}), \mathbf{z} \in \mathbb{R}^{n}$.

The advantage of Theorem 13 consists in dealing with only one DE (15), whose coefficients have unique and constant values. Moreover, inequalities (16) may be frequently fulfilled in practical studies, fact which ensures a complete answer to the $\mathrm{CWAS}_{\mathbf{v}}$ investigation.

\section{Componentwise Exponential Asymptotic Stability}

Consider the vector function:

$$
\mathbf{v}(t)=\alpha r^{t}, \alpha=\left[\alpha_{1, \ldots,}, \alpha_{n}\right]^{\prime} \in \mathbb{R}^{n}, \alpha_{i}>0, i=\overline{1, n}, 0<r<1 .
$$

Definition 3 EP $\{0\}$ of DTNUS (1) is called componentwise exponential asymptotically stable (CWEAS) if there exist a vector $\alpha \in \mathbb{R}^{n}, \alpha>0$, and a constant $0<r<1$ such that for any $\mathbf{x}\left(t_{0}\right)=\mathbf{x}_{0}$ with $\left|\mathbf{x}_{0}\right| \leq \alpha r^{t_{0}}$, the following inequality holds: $|\mathbf{x}(t)| \leq \alpha r^{t}, t \in\left[t_{0}, \infty\right)$.

Remark 8 Definition 3 can be restated in terms of Definition 2, by taking for $\mathbf{v}(t)$ the particular form given by (17).

Theorem 14 EP $\{0\}$ of DTNUS (1) is CWEAS iff the following nonlinear algebraic inequalities are compatible (have solutions $\alpha_{i}>0$, $i=\overline{1, n}, 0<r<1)$ :

$$
\begin{aligned}
& \sum_{j=1}^{n} \frac{\bar{c}_{i j} \alpha_{j}^{p_{i j}}}{\alpha_{i}} \leq r, i=\overline{1, n}, \\
& \sum_{j=1}^{n} \frac{\widetilde{c}_{i j} \alpha_{j}^{p_{i j}}}{\alpha_{i}} \leq r, i=\overline{1, n} .
\end{aligned}
$$

Proof. Necessity. It is immediate if one uses the vector function given by (17) in DIs (7-a) and (7-b) and takes $t=0$. Sufficiency. By induction for $t \in \mathbb{Z}_{+}$, one can show that functions of type (17) satisfying (18-a) and (18-b) also satisfy DIs (7-a) and (7-b).

Theorem 15 EP $\{0\}$ of DTNUS (1) is CWEAS iff the following nonlinear algebraic inequalities are compatible (have solutions $\alpha_{i}>0, i=\overline{1, n}$ ):

$$
\begin{gathered}
\sum_{j=1}^{n} \frac{\bar{c}_{i j} \alpha_{j}^{p_{i j}}}{\alpha_{i}}<1, i=\overline{1, n}, \\
\sum_{j=1}^{n} \frac{\widetilde{c}_{i j} \alpha_{j}^{p_{i j}}}{\alpha_{i}}<1, i=\overline{1, n} .
\end{gathered}
$$


Proof. This is an immediate consequence of Theorem 14 due to the fact that $0<r<1$.

Remark 9 Theorems 14 and 15 show that the CWEAS property of EP $\{0\}$ can exist even for exponents $p_{i i} \geq 2$, unlike the corresponding results of the continuous case in ([3]) requesting $p_{i i}=1$. This difference may be simply explained by comparing the general form of DTNUS (1) and the discretization of the continuous-time systems considered in ([3]), as already suggested by Remark 5 .

Conditioning the existence of CWEAS to the values of $p_{i i}$ in DTNUS (1) (as stated in Theorem 10) rises a direct question about the link between CWEAS and CWAS $_{\mathbf{v}}$.

Theorem 16 There exists a positive vector $\mathbf{v}(t)$ meeting condition (12) such that the EP $\{0\}$ of DTNUS (1) is CWA $S_{\mathbf{v}}$ iff the EP $\{0\}$ is CWEAS.

Proof. Sufficiency is obvious according to Definition 3 and Remark 8. Necessity can be proven using Theorem 8 and noticing that for at least one value $t^{*} \in\left[t_{0}, \infty\right)$ one has $v_{i}\left(t^{*}+1\right) \leq v_{i}\left(t^{*}\right), i=\overline{1, n}$ in DIs (7-a) and $(7-b)$.

The nonlinear algebraic inequalities (18-a), (18-b) and (19-a), (19-b) can be written compactly in a matrix form, using norm $\infty$, by considering the square matrices $\overline{\mathbf{M}}, \widetilde{\mathbf{M}} \in \mathbb{R}^{n \times n}$ with the following entries:

$$
\begin{aligned}
(\bar{M})_{i j} & =\bar{c}_{i j} \frac{\alpha_{j}^{p_{i j}}}{\alpha_{i}}, i, j=\overline{1, n} ; \\
(\widetilde{M})_{i j} & =\widetilde{c}_{i j} \frac{\alpha_{j}^{p_{i j}}}{\alpha_{i}}, i, j=\overline{1, n} .
\end{aligned}
$$

Theorem 17 EP $\{0\}$ of DTNUS (1) is CWEAS iff there exist $\alpha_{i}>0$, $i=\overline{1, n}$ and $0<r<1$ such that:

$$
\max \left\{\|\overline{\mathbf{M}}\|_{\infty},\|\widetilde{\mathbf{M}}\|_{\infty}\right\} \leq r .
$$

Proof. The inequalities (18-a) and (18-b) could be written, using (20-a) and $(20-b)$ in the form (21).

Theorem 18 EP $\{0\}$ of DTNUS (1) is CWEAS iff there exist $\alpha_{i}>0$, $i=\overline{1, n}$ such that:

$$
\max \left\{\|\overline{\mathbf{M}}\|_{\infty}^{\prime},\|\widetilde{\mathbf{M}}\|_{\infty}\right\}<1
$$


Proof. This is a direct consequence of Theorem 17, due to the fact that $r$ is subunitary.

Remark 10 Theorems 17 and 18 present the advantage of a more tractable formulation from the computational point of view than inequalities (18-a), (18-b) and (19-a), (19-b), respectively. Thus, the determination of $\alpha_{i}, i=\overline{1, n}$ and $r$ can be approached as a nonlinear optimization problem with adequate constraints.

As already discussed in the general case of $\mathrm{CWAS}_{\mathbf{v}}$, it might be preferable to use a sufficient condition generated from DE (15) in Theorem 13. Therefore, consider the square matrix $\widehat{\mathbf{P}} \in \mathbb{R}^{n \times n}$, with the following entries:

$$
(\widehat{P})_{i j}=\widehat{c}_{i j} \varepsilon^{p_{i j}-1}, \varepsilon>0
$$

where $\widehat{c}_{i j}, i, j=\overline{1, n}$ are defined in Theorem 13. Denote by $\lambda_{\max }(\widehat{\mathbf{P}})$ the eigenvalue of $\widehat{\mathbf{P}}$ (simple or multiple) with the largest absolute value (spectral radius).

Theorem 19 If, for a given $\varepsilon>0$, matrix $\widehat{\mathbf{P}}$ is Schur stable, then the $E P\{0\}$ of DTNUS (1) is CWEAS for some $0<\alpha_{i} \leq \varepsilon, i=\overline{1, n}$, and $\lambda_{\max }(\widehat{\mathbf{P}}) \leq r<1$.

Proof. It results from Theorem 14, by replacing all $\alpha_{i j}^{p_{i j}-1}>0, i, j=\overline{1, n}$ by their common upper bound $\varepsilon^{p_{i j}-1}$, followed by the use of Theorem 17.

The advantage of Theorem 19 consists in a quick test on the stability of matrix $\widehat{\mathbf{P}}$ which depends on a single parameter $\varepsilon>0$.

Remark 11 According to Theorem 13, whenever inequalities 16 are satisfied, the existence of a positive $\varepsilon>0$ for which matrix $\widehat{\mathbf{P}}$ is Schur stable represents a necessary and sufficient condition for EP $\{0\}$ of DTNUS (1) to be CWEAS.

\section{Conclusions}

The exploitation of the FI concepts in analyzing the dynamics of DTNUS (1) gives the possibility to individually monitor each variable of the state vector, yielding a characterization in terms of difference inequalities (Theorems 1,2,3). The presence of the interval-type coefficients, although increasing the complexity of the results, responds to 
a key problem, frequently encountered in practice, when inherent errors affect the accuracy of the model. The FI results open the way to a componentwise approach to the stability by developing specialized instruments for CWAS analysis (Theorems 8, 9, 12, 13) and CWEAS analysis (Theorems 14, 15, 17, 18, 19), respectively.

Despite a rough similarity between the difference equation of DTNUS (1) and the differential equation describing the class of continuous-time systems considered in ([3]), expecting strictly analogue properties for the solutions in the two cases is meaningless, as reflected by Remarks 1, 2, 5, 9. These remarks are able to outline the importance and the novelty of our study, because the discrete-time dynamics we are dealing with is not the result of uniformly sampling the continuous-time dynamics analyzed in ([3]) (which would lead to a difference equation with unavoidable linear terms, generated by the first order approximation of the derivatives in the differential equation).

\section{References}

[1] Hmamed, A. (1997). "Componentwise stability of 1-D and 2-D linear discrete systems", Automatica, 33(9), pp. 1759-1762.

[2] Pastravanu, O. and Voicu, M. (1999). "Flow invariant rectangular sets and componentwise asymptotic stability of interval matrix systems", Proc. of the 5-th European Control Conference ECC'99, Kalsruhe, CD-ROM.

[3] Pastravanu, O. and Voicu, M. (2001). "Flow invariance in exploring stability of a class of nonlinear uncertain systems", Proc. of the 6-th European Control Conference ECC'01, Porto, CD-ROM.

[4] Pavel, H. N. (1984). Differential Equations: Flow Invariance and Applications. Research Notes in Mathematics, No. 113, Pitman, Boston.

[5] Voicu, M. (1984a). "Free Response Characterization via flow-invariance", Prep. of the 9-th World Congress of IFAC, Budapest, Vol. 5, pp. 12-17.

[6] Voicu, M. (1984b). "Componentwise asymptotic stability of linear constant dynamical systems", IEEE Trans. Automatic Control, 29(10), pp. 937-939.

[7] Voicu, M. (1987). "On the application of the flow-invariance method in the control theory and design", Prep. of th 10-th World Congress of IFAC, Munich, Vol. 8, pp. 364-369. 